\title{
Hydrodynamic Maxwell demon in granular systems
}

\author{
J. Javier Brey, F. Moreno, R. García-Rojo, and M. J. Ruiz-Montero \\ Física Teórica, Universidad de Sevilla, Apartado de Correos 1065, 41080 Sevilla, Spain
}

(Received 19 April 2001; published 18 December 2001)

\begin{abstract}
Spontaneous symmetry breaking in a vibrated system confined into two connected compartments in the absence of external fields is reported. For a small number of particles, the grains are equipartitioned, but if it is increased beyond a critical value, the number of particles in each of the compartments becomes different in the steady state, and the number of particles in one of the compartments decreases monotonically tending to a given value. This phase transition is accurately described by the hydrodynamic equations for a granular gas. The relationship with previous phenomena of phase separation in vibrofluidized granular materials is discussed.

DOI: 10.1103/PhysRevE.65.011305

PACS number(s): 45.70.Mg, 05.70.Ln, 81.05.Rm
\end{abstract}

Granular materials exhibit a series of fascinating effects due to their character of dissipative nonequilibrium systems [1]. Besides, given the macroscopic size of the grains, these effects are easy to observe experimentally in many cases. Although real granular media are composed by rough particles interacting in a very complex way, it has been shown that many of the characteristic features of their behavior can be described by means of simple mechanical models, such as a system of smooth inelastic hard spheres.

One of the main qualitative differences between ordinary molecular systems and granular media is the strong tendency of the latter to develop spatial inhomogeneities. In fact, inelasticity and spatial gradients are coupled in any stationary state of a granular fluid [2]. This property is inherent to the dissipation of energy in collisions and, in principle, it is not directly associated to the presence of instabilities in the system. On the other hand, granular flows also present the socalled clustering effect, where regions of very high density coexist with very dilute ones [3-5]. Clustering was first observed in molecular-dynamics simulations of freely evolving granular fluids, and Goldhirsch and Zanetti [3] showed that it can be explained by means of a hydrodynamic description of the granular gas. In this context, it appears as a longwavelength hydrodynamic instability induced by a fluctuation of the transversal component of the velocity, i.e., the shear mode [6]. Recently, clustering has been observed experimentally in driven granular systems $[7,8]$.

Several years ago, Schlichting and Nordmeier [9] reported the results of a simple experiment with grains indicating a behavior fundamentally different from that of a molecular system. The experiment has been reproduced in Ref. [10] and similar results were found. An open box is filled with particles of a granular material, which are brought into a fluidized state by shaking the system vertically. The box is separated into two compartments of the same section by a vertical wall of a certain height. For strong shaking, the particles distribute themselves equally to both sides of the wall. Nevertheless, if the intensity of the driving is lowered below a critical value, the spatial symmetry of the system is spontaneously broken and the number of particles in one of the compartments becomes larger than in the other one. The asymmetry increases as the shaking intensity decreases. Particles in the low-density compartment have much larger en- ergy than those in the low-density section. For this reason, the above experiment is sometimes referred to as the Maxwell demon experiment.

Eggers [11] has proposed an analytical theory to explain the existence of the above asymmetric steady state. In his approach, the interior wall separating both compartments is formally extended up to infinity and a small hole at a given height is introduced. Assuming that the interchange of particles between the two compartments can be considered as an effusion process, the stationarity condition requiring that the net flux between the two compartments is null can be written down by using a simple kinetic theory for granular gases. The result is that there is a phase transition with symmetry breaking determined by a parameter that depends on the intensity of vibration, the inelasticity of collisions, and the number of monolayers of particles at rest [11]. The theoretical prediction for the bifurcation diagram compares fairly well with the results from molecular-dynamics simulations as well as with those from experiments [10].

In this paper, the existence of another spontaneous symmetry breaking in vibrated granular systems is reported. Although at first sight it looks similar to the one discussed above, both differ in several aspects. The most relevant one is that the one reported here is explained by considering the hydrodynamic stationarity conditions. This is because the symmetry breaking is observed when the linear size of the hole connecting both compartments is larger than the mean free path of the gas in its vicinity. Let us point out that in this regime, the bifurcation reported in [9-11] disappears, indicating quite dramatically the different nature of both phenomena. Besides, we consider a system in the absence of any external field. As a consequence of the above, the control parameter is different in both cases. On the other hand, it is important to note that neither of the two transitions has a characteristic length scale, being therefore clearly distinct from the clustering instability. Finally, we stress that, in the present case, the pressure is uniform and the same in both compartments, showing that a pressure gradient cannot be responsible for the symmetry breaking.

Let us start by describing the findings from moleculardynamics simulations. The simulated system consists of a two-dimensional box of width $2 S$ and height $L$ containing $N$ circular disks of mass $m$ and diameter $\sigma$. The box is sepa- 


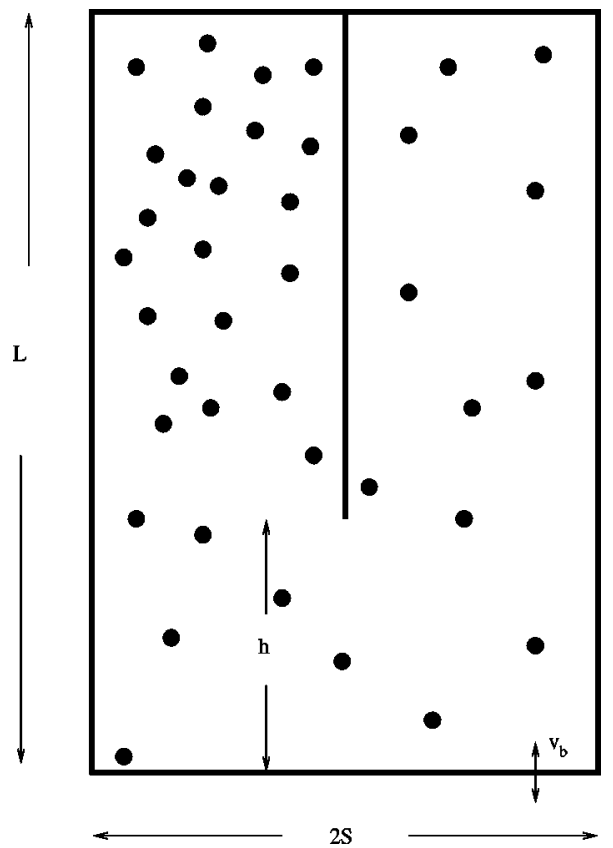

FIG. 1. Sketch of the system considered in the text. The wall at the bottom is vibrated with a sawtooth velocity profile and very small amplitude. The two sides of the box are connected by a gap of height $h$ starting at the bottom of the container.

rated into two compartments of the same width $S$ by a vertical wall starting at a height $h$ (see Fig. 1). No external field is acting on the system. The wall at the bottom is vibrated in a sawtooth way with a velocity $v_{b}$. Therefore, all particles colliding with the wall find it with that velocity [12]. Besides, the amplitude of the vibration is considered much smaller than the mean free path of the particles next to it, so that the position of the bottom can be taken as fixed. Collisions of the particles with the walls are considered as elastic, while collisions between particles are characterized by a constant coefficient of normal restitution $\alpha$. Keeping all the other parameters of the system constant, we have carried out a series of simulations changing the total number of particles $N$.

For small values of $N$ a steady state is reached in which the particles are equally divided into the two compartments. When the value of $N$ is increased beyond a critical value, the symmetry is spontaneously broken and the populations at both sides of the wall become different in the steady state. The asymmetry of the transition can be characterized by the parameter $\epsilon=\left(N-2 \bar{N}^{(l)}\right) / 2 N$, where $\bar{N}^{(l)}$ is the time-average number of particles in the left compartment in the steady state. The resulting bifurcation diagram is shown in Fig. 2, where $\epsilon$ is plotted as a function of $\xi_{m}$, which is a dimensionless quantity proportional to the total number of particles $N$ to be defined later on. The molecular-dynamics data in Fig. 2 correspond to $L=140 \sigma$ and $S=50 \sigma$. The size $h$ of the hole has been chosen to be of the order of twice the mean free path of the gas next to it. A typical value in the reported simulations is $h=50 \sigma$. Results for two different values of $\alpha$, namely 0.95 and 0.9 , have been included. The data are seen to collapse on the same curve when plotted as a function of

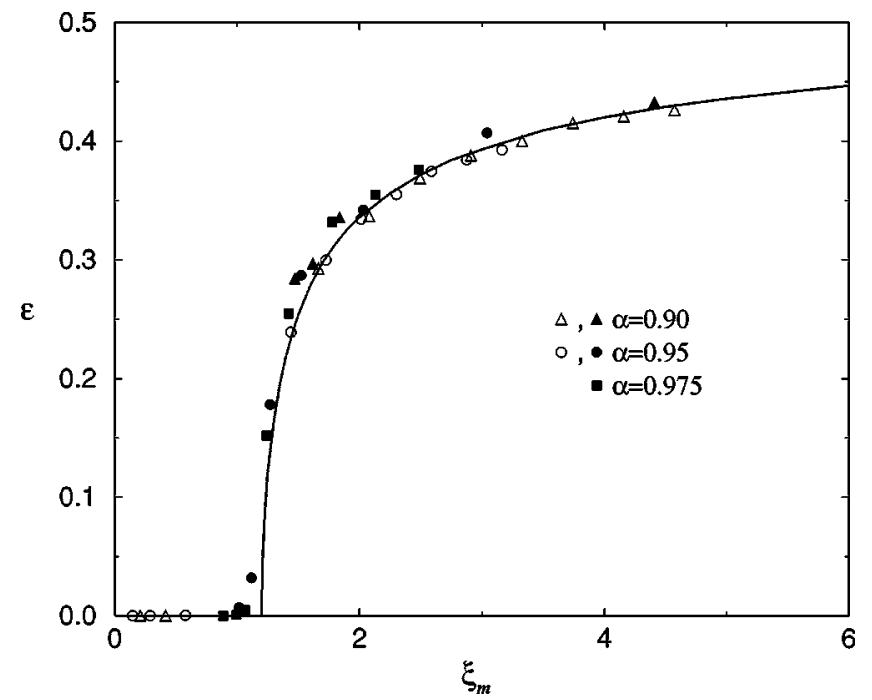

FIG. 2. Bifurcation diagram showing the asymmetry of the number of particles $\epsilon$ as a function of the dimensionless parameter $\xi_{m}$ defined in the main text. The open symbols are from moleculardynamics simulations, and the filled ones from DSMC. The full line is the theoretical prediction from the model developed in the paper.

$\xi_{m}$. Moreover, we have verified that the bifurcation diagram is not altered by modifying the velocity of the wall $v_{b}$, as long as it is large enough to keep the granular system fluidized. Similar results have been obtained by numerical solution of the inelastic Boltzmann equation by means of the direct simulation Monte Carlo (DSMC) method [13]. Some of them have been included in Fig. 2 and also fit in the same diagram.

In order to explain what is observed in the simulations, we have used the hydrodynamic description of a vibrated granular gas of hard disks $(d=2)$ or spheres $(d=3)$. Let us consider a dilute granular gas confined between a vibrating wall at $x=0$ and a reflecting one at $x=L$, and assume there are gradients only in the $x$ direction. It is convenient to introduce a dimensionless space variable $\xi$ by

$$
\xi=\sqrt{a(\alpha)} \int_{0}^{x} \frac{d x^{\prime}}{\lambda\left(x^{\prime}\right)},
$$

where $\lambda=\left(C \sigma^{d-1} n\right)^{-1}$ is the local mean free path, $n(x)$ being the local number density, and $C=2 \sqrt{2}$ for $d=2$ and $C=\pi \sqrt{2}$ for $d=3$. Moreover,

$$
a(\alpha)=\frac{32(d-1) \pi^{d-1} \zeta^{*}(\alpha)}{(d+3)^{3} C^{2} \Gamma(d / 2)^{2}\left[\kappa^{*}(\alpha)-\mu^{*}(\alpha)\right]} .
$$

In this expression, $\kappa^{*}(\alpha)$ and $\mu^{*}(\alpha)$ are rather involved functions of $\alpha$ describing the heat flux in a dilute granular gas [14]. For small inelasticity ( $\alpha$ close to unity), the former can be approximated by unity and the latter can be neglected. The function $\zeta^{*}(\alpha)$ is associated to the cooling rate due to the energy dissipation in collisions and near the elastic limit, $\quad \zeta^{*}(\alpha) \simeq(2+d)(1-\alpha) / 2 d$. For $x=L, \quad \xi=\xi_{m}$ $\equiv \sqrt{a(\alpha)} C \sigma^{d-1} N_{x}$, with $N_{x}$ being the number of particles 


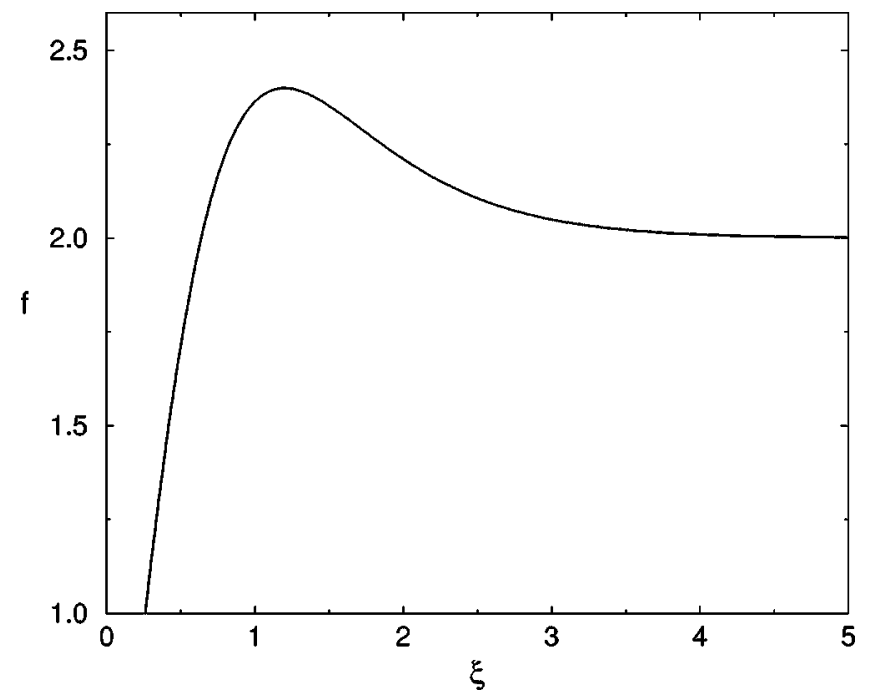

FIG. 3. Function $f(\xi)$ defined in Eq. (6).

per unit of wall section. This is the quantity (applied to the whole system) used in the horizontal axis in Fig. 2.

In the $\xi$ scale, the temperature profile $T(\xi)$ and the (uniform) pressure $p$ are given by [15]

$$
\begin{gathered}
T(\xi)=T_{0}\left[\frac{\cosh \left(\xi_{m}-\xi\right)}{\cosh \xi_{m}}\right]^{2}, \\
p=\frac{k_{B} T_{0}}{4 C \sigma^{d-1} L \sqrt{a(\alpha)}} \xi_{m}+\sinh \left(2 \xi_{m}\right) \\
\cosh ^{2} \xi_{m}
\end{gathered}
$$

respectively. Here $k_{B}$ is the Boltzmann constant, taken often as unity in the granular literature, and $T_{0}$ denotes the temperature of the granular gas next to the vibrating wall. The above expressions are closed with the equation of state $p$ $=n k_{B} T$.

Next we model the steady state reached by the system illustrated in Fig. 1 by considering two independent compartments, sharing only a thin layer of granular gas at temperature $T_{0}$ located at the bottom of the container, formally at $x$ $=0$. The steady state of the gas in each of the two compartments is accurately described by Eqs. (3) and (4). The hydrodynamic momentum equation applied to the whole system requires the pressure to be the same in both compartments, and use of Eq. (4) yields

$$
f\left(\xi_{m}^{(l)}\right)=f\left(\xi_{m}^{(r)}\right)
$$

where

$$
f(\xi)=\frac{2 \xi+\sinh (2 \xi)}{\cosh ^{2} \xi}
$$

and the indexes $l$ and $r$ refer to the left and right compartment, respectively. The conservation of the total number of particles implies that

$$
\xi_{m}^{(l)}+\xi_{m}^{(r)}=2 \xi_{m}=2 \sqrt{a(\alpha)} C \sigma^{d-1} N_{x}
$$

(a)

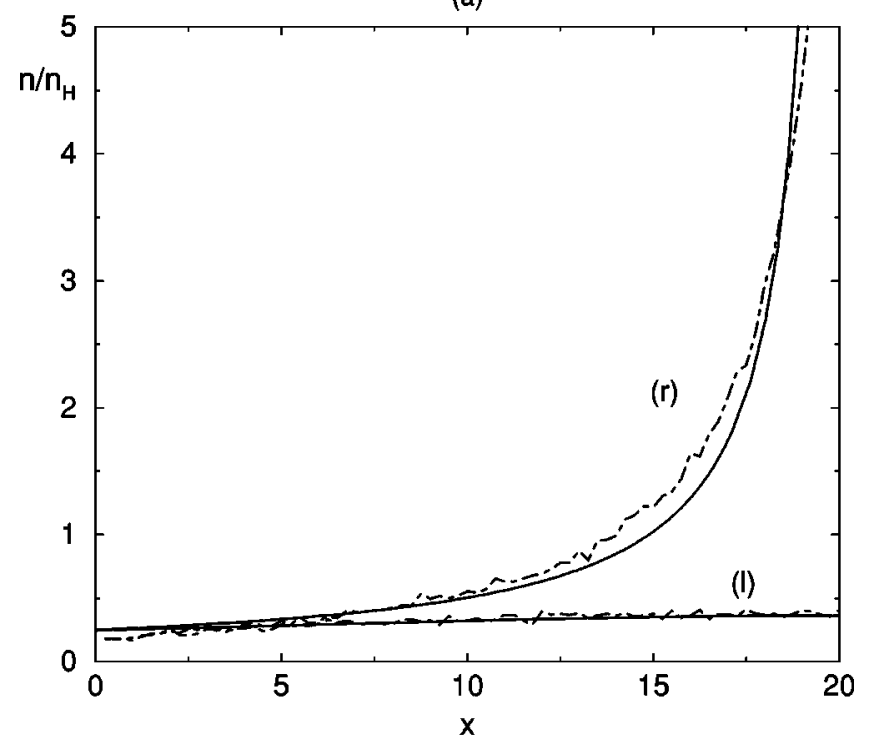

(b)

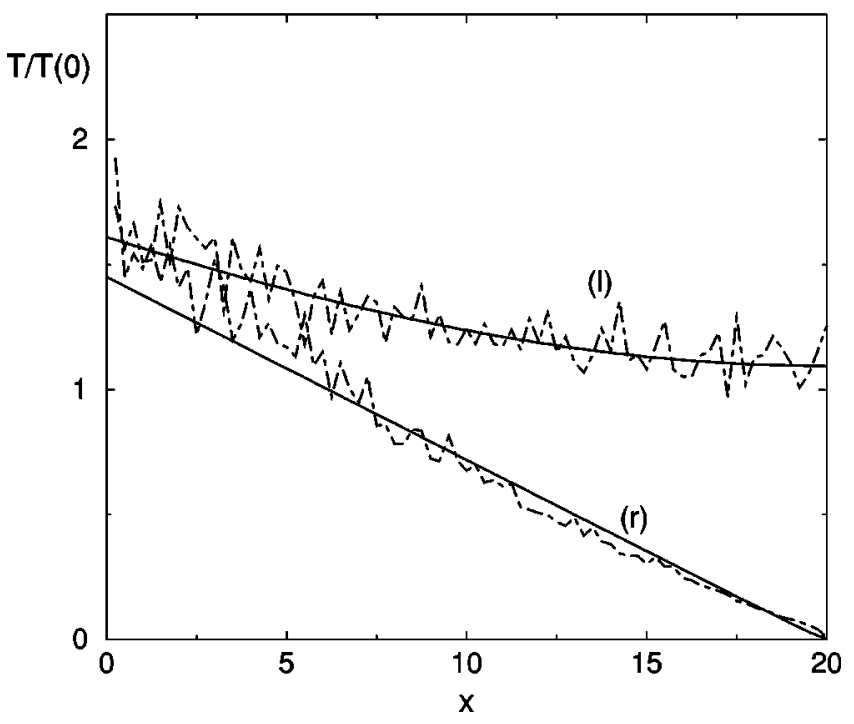

FIG. 4. Density (a) and temperature (b) profiles in each of the two compartments $[(l)$ and $(r)]$, in the steady state for a system with $\xi_{m}=2.03$. The dashed lines are from DSMC and the solid lines the theoretical prediction derived in the text. The coordinate $x$ is measured in units of the homogeneous mean free path, the density is scaled with its homogeneous value, $n_{H}$, and the temperature with the initial one, $T(0)$.

Note that now $N_{x}=N / 2 S$. Of course, Eq. (5) always has the trivial solution $\xi_{m}^{(l)}=\xi_{m}^{(r)}=\xi_{m}$, corresponding to the symmetric state with the same number of particles in both compartments. In order to investigate whether there is another solution, one has to analyze the function $f(\xi)$ that is plotted in Fig. 3. It has a maximum at $\xi=\xi_{0}$, where $\xi_{0}$ is the root of the equation $\xi_{0} \tanh \xi_{0}=1$. Numerically it is found that $\xi_{0}$ $\simeq 1.20$. For $\xi_{m}<\xi_{0}$, there is only the symmetric solution, while for any $\xi_{m}>\xi_{0}$, there are in addition two values $\xi_{m}^{(l)}$ and $\xi_{m}^{(r)}, \xi_{m}^{(l)} \neq \xi_{m}^{(r)}$, verifying Eqs. (5) and (7). The asymmetry of the two populations, as measured by $\epsilon$, increases very fast as the total number of particles increases beyond the 
critical value $N_{0}$ determined by $\xi_{0}=\sqrt{a(\alpha)} C \sigma^{d-1} N_{0} / 2 S$. Even more, the steady number of particles in one of the compartments decreases when more particles are added to the system, and approaches a fixed value corresponding to a very low density. This unexpected behavior, indicating the tendency of the system to keep a low-density region, placing all the surplus particles in the high-density compartment, is confirmed by the simulation results. The solid line in Fig. 2 shows the solutions of Eq. (5) as a function of $\xi_{m}$. Note that, plotted in this way, the $\alpha$ dependence of the theoretical prediction has been scaled out. A fairly good agreement between theory and numerical simulations is observed. Just above the critical point, i.e., for $0<\xi_{m}-\xi_{0} \ll 1$, the asymmetric solutions are described by

$$
\epsilon= \pm\left[A_{0}\left(\xi_{m}-\xi_{0}\right)\right]^{1 / 2},
$$

with $A_{0} \simeq 0.31$, indicating a critical behavior described by the exponent $\beta=1 / 2$. This corresponds to a free energy of the classical Landau form for a second-order phase transition.

We have also investigated the hydrodynamic profiles in each of the two compartments. As an example, in Fig. 4 we compare the theoretical predictions for the temperature and density profiles with the results obtained by the DSMC method in one of the cases. Although similar profiles are obtained from molecular-dynamics simulations [16], the DSMC has the advantage of avoiding the excluded volume effects, which are not accounted for by the hydrodynamic description we are employing, based on the Boltzmann equation. The figure corresponds to a system of disks with $\alpha$ $=0.95$, and $m$ has been used as the unit of mass, the homogeneous mean free path, $\lambda_{H}=L\left(2 \sqrt{2} N_{x} \sigma\right)^{-1}$ as the unit of length, and the unit of temperature is defined by the average kinetic energy of the initial state, namely $2 k_{B} T(0)=1$. In these units, the values of the parameters in Fig. 4 are $L$ $=20, h=7.5$, and $v_{b}=0.4$, leading to $\xi_{m}=2.03$. The theoretical curves for the temperature have been constructed by using $T_{0}^{(l)}$ and $T_{0}^{(r)}$ as two fitting parameters describing the temperature of the gas next to the vibrating wall in the left and right compartments, respectively. Although in the simplified theory we have developed we considered $T_{0}^{(l)}=T_{0}^{(r)}$ $=T_{0}$, and neglected the size of the common region next to the vibrating wall, the simulation results indicate that the state of this boundary layer is, in fact, quite complicated, as expected. The values of the measured hydrodynamic variables there do not correspond to the extrapolation from the bulk of the compartments. On the other hand, the theoretical density profiles do not involve any fitting parameter. The shape of the profiles in Fig. 4 shows that the system segregates into two different "phases." While in one of the compartments the fluid is dense and cold, in the other it is dilute and hot. As discussed above, the situation looks as if there were a Maxwell demon determining that the number of particles in one of the compartments remains very low. The fairly good agreement between theory and simulation shown in the figure supports the accuracy of the model.

In summary, we have identified a spontaneous symmetry breaking in a vibrated granular system in the absence of external fields. The phenomenon can be understood on the basis of the hydrodynamic equations that, once again, appear as a very valuable tool to describe granular fluids, at least in the dilute limit.

A physically relevant question asks why the system actually prefers the asymmetric state to the symmetric one. Addressing this issue requires a detailed analysis of the stability of the steady state, which will be considered elsewhere. Here we only mention that from a simple energy balance, it follows that the power dissipated in collisions, for a given value of the velocity of the vibrating wall, is larger in the asymmetric stable state than in the symmetric one.

We acknowledge financial support from the Dirección General de Investigación Científica y Técnica (Spain) through Grant No. PB98-1124.
[1] H.M. Jaeger, S.R. Nagel, and R.P. Behringer, Rev. Mod. Phys. 68, 1259 (1996).

[2] P.K. Haff, J. Fluid Mech. 134, 401 (1983).

[3] I. Goldhirsch and G. Zanetti, Phys. Rev. Lett. 70, 1619 (1993).

[4] I. Goldhirsch, M.L. Tan, and G. Zanetti, J. Sci. Comput. 8, 1 (1993).

[5] S. McNamara and W.R. Young, Phys. Rev. E 50, R28 (1994); 53, 5089 (1996).

[6] J.J. Brey, M.J. Ruiz-Montero, and D. Cubero, Phys. Rev. E 60, 3150 (1999).

[7] W. Losert, D.G.W. Cooper, and J.P. Gollub, Phys. Rev. E 59, 5855 (1999).

[8] J.S. Olafsen and J.S. Urbach, Phys. Rev. Lett. 81, 4369 (1998); Phys. Rev. E 60, R2468 (1999).

[9] H.J. Schlichting and V. Nordmeier, Math. Naturwiss. Unterr.

\section{9, 323 (1996).}

[10] K. van der Weele, D. van der Meer, M. Versluis, and D. Lohse, Europhys. Lett. 53, 328 (2001).

[11] J. Eggers, Phys. Rev. Lett. 83, 5322 (1999).

[12] S. McNamara and J-L. Barrat, Phys. Rev. E 55, 7767 (1997).

[13] G. Bird, Molecular Gas Dynamics and the Direct Simulation of Gas Flows (Clarendon Press, Oxford, 1994).

[14] J.J. Brey and D. Cubero, in Granular Gases, edited by T. Pöschel and S. Luding, Lecture Notes in Physics, Vol. 59 (Springer-Verlag, Berlin, 2001).

[15] J.J. Brey, M.J. Ruiz-Montero, and F. Moreno, Phys. Rev. E 62, 5339 (2000).

[16] J.J. Brey, M.J. Ruiz-Montero, F. Moreno, and R. García-Rojo (unpublished). 